\title{
A Refined ProTide Route to Uprifosbuvir
}

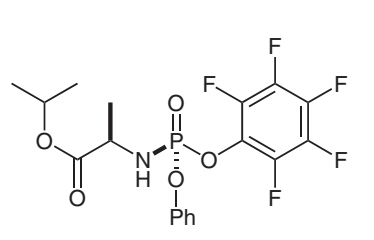

A (1.0 equiv)

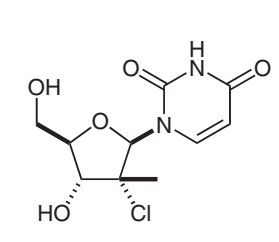

B (1.2 equiv)

$\mathrm{Me}_{2} \mathrm{AlCl}(0.5$ equiv) 2,6-Iutidine (1.25 equiv) $\operatorname{THF}(15 \mathrm{Vol}), 35^{\circ} \mathrm{C}, 15 \mathrm{~h}$ $\underset{\text { cryst ex } i-\mathrm{PrOH}-\text { heptane }}{\stackrel{95 \% \text { conversion; }}{\longrightarrow}}$ $81 \%(5.42 \mathrm{~mol}$ scale $)$

C/D > 99:1<smiles>CC(C)OC(=O)C(C)N</smiles><smiles>CNP(=O)(OC[C@H]1OC(n2ccc(=O)[nH]c2=O)[C@](C)(Cl)[C@@H]1O)Oc1ccccc1</smiles>

C (Uprifosbuvir) $\mathrm{dr}>$ 99:1 $+$

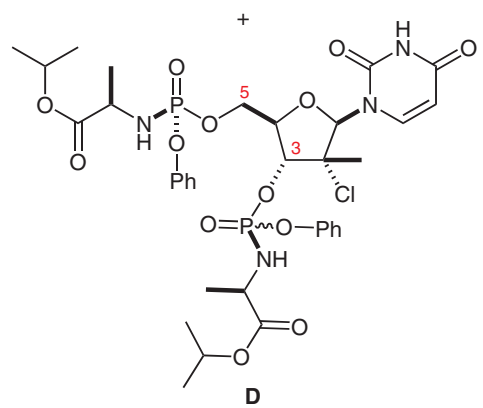

Category

Synthesis of Natural

Products and

Potential Drugs

Key words

uprifosbuvir

hepatitis C

MK-3682

phosphorylation

prodrug

nucleotides
Significance: The hepatitis $\mathrm{C}$ virus (HCV) has infected 170 million people worldwide. Over 70 million have succumbed to chronic hepatitis $C$ that can lead to a spectrum of conditions affecting the liver, ranging from inflammation to cirrhosis and cancer. Uprifosbuvir (MK-3682) is an NS5B RNA polymerase inhibitor that was of interest as a combination therapy for the treatment of HCV infections. It is a nucleoside-based prodrug that utilizes the $5^{\prime}$ aryloxyphosphoramidate or ProTide moiety $\mathbf{A}$ to enhance cellular permeability and phosphorylation rates. Over 20 forms of uprifosbuvir have been identified, some showing dramatically reduced bioavailability.
Comment: A major improvement in the multikilogram-scale synthesis of $C$ entailed the reaction of phosphoramidate $\mathbf{A}$ ( 1.0 equiv) with nucleoside $\mathbf{B}$ (1.2 equiv) promoted by dimethylaluminum chloride ( 0.5 equiv) and 2,6-lutidine ( 1.25 equiv) in THF at $35^{\circ} \mathrm{C}$. Under these precisely defined conditions, uprifosbuvir was isolated in $81 \%$ yield with >100:1 diastereoselectivity at the phosphorus stereocenter and >100:1 selectivity for the 5 '-mono phosphorylation product $\mathrm{C}$ over the undesired bisphosphorylation side products $\mathbf{D}$. A small increase in the reaction temperature led to a significant increase in the formation of cyclic phosphoramidate impurity $\mathbf{E}$. Techniques and apparatus are described to safely handle neat pyrophoric dimethylaluminum chloride. 